\title{
CATEGORICAL UNIVERSALITY OF REGULAR DOUBLE P-ALGEBRAS
}

\author{
by V. KOUBEK $\dagger$ and J. SICHLER $\dagger$
}

(Received 20 April, 1989)

1. Introduction. An algebra $A=\left(L ; \vee, \wedge,{ }^{*},{ }^{+}, 0,1\right)$ of type $(2,2,1,1,0,0)$ is a double p-algebra if $(L ; \vee, \wedge, 0,1)$ is a $(0,1)$-lattice in which ${ }^{*}$ and ${ }^{+}$are unary operations of pseudocomplementation and dual pseudocomplementation determined by the respective requirements that $x \leqslant a^{*}$ be equivalent to $x \wedge a=0$, and that $x \geqslant a^{+}$if and only if $x \vee a=1$.

For any double p-algebra $A$, the collection $\Phi$ of all pairs $(a, b) \in A^{2}$ which satisfy $a^{*}=b^{*}$ and $a^{+}=b^{+}$is a congruence, called the determination congruence of $A$. An algebra is regular if the determination congruence of $A$ is the diagonal of $A^{2}$; according to Varlet [12], the class $\mathbf{R}$ of all regular distributive double $\mathrm{p}$-algebras is a variety determined by the identity equivalent to $x \wedge x^{+} \leqslant y \vee y^{*}$. The variety $\mathbf{R}$ is congruence permutable, congruence regular, and all compact congruences of its members are principal (cf. [12], Katriňák [5], or Adams and Beazer [2]).

For any element $a$ of a double p-algebra $A$ and for any integer $n \geqslant 0$, define $a^{n(*+)}$ recursively by $a^{0(*+)}=a$, and $a^{(n+1)(*+)}=a^{n(*+) *+}$. Algebras in the variety $\mathbf{B}_{n}$ defined by the identity $x^{(n+1)(*+)}=x^{n(*+)}$ are said to be of range $n$; since $b \in A$ is complemented if and only if $b^{*+}=b$, it follows, for instance that $\mathbf{B}_{0}$ is the variety of Boolean algebras (cf. [6]). Whenever a variety $\mathbf{V}$ is contained in $\mathbf{B}_{n}$ for some $n \geqslant 0$, we say that $\mathbf{V}$ is of bounded range.

A category $\mathbf{C}$ is universal if any full category of algebras is isomorphic to a full subcategory of $\mathbf{C}$. For every monoid $M$, any universal category thus contains a proper class of pairwise non-isomorphic objects $X$ whose endomorphism monoid $\operatorname{End}(X)$ is isomorphic to $M$ (see [4] or Pultr and Trnková [11]); consequently, any universal category of algebras must contain arbitrarily large objects representing $M$ in this manner.

We present the following two results.

THEOREM 1.1. The variety $\mathbf{R}$ of regular double p-algebras is universal.

THEOREM 1.2. No universal subvariety $\mathbf{V}$ of $\mathbf{R}$ is of bounded range.

The question below arises naturally from these two theorems.

Problem. Characterize universal varieties of regular double p-algebras.

It is perhaps interesting to note two related, if contrasting, results of [7]: the first states that every group occurs as the full automorphism group of a regular double p-algebra of range two, while the second shows that there exist (non-regular) finitely generated universal varieties of distributive double p-algebras of range three.

Theorem 1.1 also provides the first natural example of a universal variety which is both arithmetical and congruence regular, thus contributing towards Ervin Fried's inquiry about the effect congruence properties of varieties may have on categorical universality. This example can be reinterpreted in the variety of double Heyting algebras (cf. Adams and Beazer [1] and Theorem 4.5 below).

† The support of the NSERC is gratefully acknowledged.

Glasgow Math. J. 32 (1990) 329-340. 
The underlying lattice of any regular double p-algebra is distributive (Katriňák [5]), and this enables us to use Priestley's duality throughout the paper; this duality is briefly discussed in the next section. Theorem 1.2 is demonstrated in Section 3, while the proof of Theorem 1.1 constitutes the concluding section.

2. Priestley spaces. A triple $Q=(X, \tau, \leqslant)$ is an ordered topological space whenever $(X, \leqslant)$ is a poset and $(X, \tau)$ is a topological space.

For any $Z \subseteq X$ define

$$
(Z]=\{x \in X: \exists z \in Z \quad x \leqslant z\} \text { and }[Z)=\{x \in X: \exists z \in Z \quad z \leqslant x\}
$$

a set $Z$ is decreasing if $(Z]=Z$, increasing if $[Z)=Z$, and clopen if it is both $\tau$-closed and $\tau$-open.

An ordered topological space $Q=(X, \tau, \leqslant)$ is totally order disconnected if its clopen decreasing sets create its order in the sense that, for any $x, y \in X$ such that $x \neq y$ there exists a clopen decreasing set $Y \subseteq X$ such that $y \in Y$ and $x \in X \backslash Y$. Any compact totally order disconnected ordered topological space is called a Priestley space. Any such space is a compact Hausdorff space with a basis formed by all clopen convex sets and their complements. It is also easily seen that, in a Priestley space $Q$, the sets $(Y]$ and $[Y)$ are closed for any closed subset $Y$ of $Q$.

For any distributive $(0,1)$-lattice $L$, let $P(L)=(F(L), \tau, \leqslant)$ be an ordered topological space in which $(F(L), \leqslant)$ is the set $F(L)$ of all prime filters of $L$ ordered by the inverse inclusion, and such that all sets $\{x \in F(L): A \in x\},\{x \in F(L): A \notin x\}$ with $A \in L$ form an open subbasis of $\tau$. The space $P(L)$ is totally order disconnected and compact, see Priestley [8].

For any lattice $(0,1)$-homomorphism $f: L \rightarrow L^{\prime}$, the preimage $f^{-1}(x)$ of any $x \in P\left(L^{\prime}\right)$ is a prime filter of $L$, and the mapping $P(f): P\left(L^{\prime}\right) \rightarrow P(L)$ defined by $P(f)(x)=f^{-1}(x)$ preserves the order and is continuous. This gives rise to a contravariant functor $\boldsymbol{P}: \mathbf{D} \rightarrow \mathbf{P}$ of the category $\mathbf{D}$ of all $(0,1)$-homomorphisms of distributive $(0,1)$-lattices into the category $\mathbf{P}$ of all continuous order preserving maps of Priestley spaces.

When inclusion-ordered, the set $D(P)$ of all clopen decreasing subsets of any Priestley space $P$ is a distributive $(0,1)$-lattice, and the inverse-image mapping $g^{-1}$ of any P-morphism $g: P^{\prime} \rightarrow P$ restricts to a lattice $(0,1)$-homomorphism $D(g): D(P) \rightarrow D\left(P^{\prime}\right)$. This defines a contravariant functor $D: \mathbf{P} \rightarrow \mathbf{D}$.

Priestley's duality can now be described as follows.

THeorem 2.1. (Priestley [8], [9]). The composites $P \circ D$ and $D \circ P$ are naturally equivalent to the identity functors of their respective domains; the category $\mathbf{D}$ is thus dually isomorphic to $\mathbf{P}$.

The claim below gives a useful separation property of all Priestley spaces.

Proposition 2.2. If $P=(X, \tau, \leqslant)$ is a Priestley space and $Y, Z \subseteq X$ disjoint closed sets, then there exists a clopen set $A \subseteq X$ such that $Z \subseteq A$ and $Y \subseteq X \backslash A$; if, in addition, $Y \cap(Z]=\varnothing$ then $A$ may be chosen amongst clopen decreasing sets of $P$.

For any ordered topological space $(X, \tau, \leqslant)$, let $\operatorname{Min}(X)$ and $\operatorname{Max}(X)$ respectively denote the sets of all minimal or maximal elements of the poset $(X, \leqslant)$. For any $Y \subseteq X$ 
define $\operatorname{Min}(Y)=(Y] \cap \operatorname{Min}(X), \operatorname{Max}(Y)=[Y) \cap \operatorname{Max}(X)$, and $\operatorname{Ext}(Y)=\operatorname{Min}(Y) \cup \operatorname{Max}(Y)$. We write $\operatorname{Min}(x)$ instead of $\operatorname{Min}(\{x\})$, and note that, in any Priestley space, the sets $\operatorname{Min}(x)$ and $\operatorname{Max}(x)$ are nonvoid for every $x \in X$.

The following theorem characterizes Priestley spaces forming the category dual to that of distributive double p-algebras (cf. Priestley [10]).

Theorem 2.3. If $P: \mathbf{D} \rightarrow \mathbf{P}$ is the functor assigning Priestley spaces to distributive $(0,1)$-lattices and if $f: L \rightarrow L^{\prime}$ is a lattice $(0,1)$-homomorphism then

(a) $L$ is a distributive double $p$-algebra if and only if $(Y]$ is clopen for any clopen increasing subset $Y$ of $P(L)$ and $[Z)$ is clopen for any clopen decreasing subset $Z$ of $P(L)$, and if this is the case then $A^{*}=P(L) \backslash[A)$ and $A^{+}=(P(L) \backslash A]$ for any clopen decreasing set $A \subseteq P(L)$;

(b) $f$ is a homomorphism of distributive double $p$-algebras if and only if $P(f)(\operatorname{Min}(x))=\operatorname{Min}(P(f)(x))$ and $P(f)(\operatorname{Max}(x))=\operatorname{Max}(P(f)(x))$ for every $x \in P\left(L^{\prime}\right)$;

(c) if $L$ is a distributive double $p$-algebra then the sets $\operatorname{Min}(P(L))$ and $\operatorname{Max}(P(L))$ are closed.

Throughout the paper, any Priestley space satisfying (a) will be called a $d p$-space, and any continuous order preserving mapping for which (b) holds will be a dp-map.

Elements $x, y$ of a poset $(X, \leqslant)$ are connected if there exists a sequence $x=$ $x_{0}, x_{1}, \ldots, x_{n}=y$ such that $x_{i}$ is comparable to $x_{i+1}$ for all $i=0, \ldots, n-1$. Classes of the equivalence formed by all pairs of connected elements, called components of $(X, \leqslant)$, are maximal connected subsets of $(X, \leqslant)$. A connected poset $(Y, \leqslant)$ has diameter $m$ whenever $m$ is the least integer such that any $x, y \in Y$ can be connected by such a sequence with $n \leqslant m$; otherwise the diameter of $(Y, \leqslant)$ is infinite. Whether finite or infinite, the diameter of any component $C$ coincides with the diameter of $\operatorname{Ext}(C)$.

Let $P=(X, \tau, \leqslant)$ be a dp-space. For any subset $S$ of $X$ define $D_{0}(S)=S$ and, for every $k \geqslant 0$, recursively set $D_{k+1}(S)=\left(\left[D_{k}(S)\right)\right]$; we write $D_{k}(x)$ instead of $D_{k}(\{x\})$. Clearly, $y \in D_{k}(x)$ is equivalent to $x \in D_{k}(y)$ for any $k \geqslant 0$. Observe also that $D_{k}(S) \subseteq$ $D_{k+1}(S)$ for all $k \geqslant 0$, and that the component $C(x)$ containing $x \in X$ is the union of sets $D_{k}(x)$ for $k \geqslant 0$. Furthermore, noting that $2.3\left(\right.$ a) implies that $A^{*+}=([A)]$ for any clopen decreasing set $A \subseteq X$, we conclude that $D_{k}(A)=A^{k(*+)}$ for all $k \geqslant 0$. Therefore the open set $\bigcup\left\{A^{k(*+)}: k \geqslant 0\right\}$ contains exactly those components of $P$ which intersect $A$. From $X \backslash A^{+*}=[(X \backslash A])$, it similarly follows that the closed set $\bigcap\left\{A^{k(+*)}: k \geqslant 0\right\}$ is the union of all those components of $P$ which are entirely contained in $A$.

Proposition 2.4. The following are equivalent for any variety $\mathbf{V}$ of distributive double p-algebras:

(i) V satisfies the identity $x^{(n+1)(*+)}=x^{n(*+)}$ for some $n \geqslant 0$,

(ii) there is a common finite bound on the diameters of all order components of any $P(L)$ with $L \in \mathbf{V}$,

(iii) all order components of every $P(L)$ with $L \in \mathbf{V}$ are closed.

Proof. (i) $\Rightarrow$ (ii). Assume that $x$ and $y$ are distinct minimal elements of a component $C$ of $P(L)$, and let $k$ be the least integer such that $x \in D_{k}(y)$. Then $k \geqslant 1$, and $x$ does not lie in the closed decreasing set $D_{k-1}(y)$. By 2.2 , there exists a clopen decreasing set $B \supseteq D_{k-1}(y)$ such that $x \in P(L) \backslash B$. Hence the set $A=P(L) \backslash[B)$ is disjoint with $D_{k-1}(y)$, 
and $x \in A$. From $y \in D_{k}(x)$, it immediately follows that $y \in A^{k(*+)}$, while the definition of $A$ implies that $y \in A^{m(*+)}$ only when $m \geqslant k$. Thus the diameter of $C$ is at most $2 n$.

(ii) $\Rightarrow$ (iii). If a component $C(x)$ has a finite diameter then $C(x)=D_{k}(x)$ for some $k \geqslant 0$, and the set $D_{k}(x)$ is closed.

(iii) $\Rightarrow$ (i). Assume that (i) fails; so that, for every $n \geqslant 0$, there exists an algebra $L_{n} \in \mathrm{V}$ and some $A_{n} \in L_{n}$ for which $A_{n}^{(n+1)(*+)}>A_{n}^{n(*+)}$. The product $L=\Pi\left(L_{n}: n \geqslant 0\right)$ then contains the sequence $A=\left(A_{n}: n \geqslant 0\right)$ such that $A^{(n+1)(*+)}>A^{n(*+)}$ for all $n \geqslant 1$.

Consider $A$ as a clopen decreasing subset of $P(L)$ and define $B_{k}=A^{(k-1)(*+) *} \cap$ $A^{k(*+)}$ for every $k \geqslant 1$. If $B_{k}=\varnothing$ then $D=A^{(k-1)(*+) *}$ satisfies $D \cap D^{+}=\varnothing$; so that $D^{+} \leqslant D^{*}$ by the definition of the pseudocomplement. Thus $D^{*}=D^{+}$since $D^{*} \leqslant D^{+}$ always holds. Hence $D$ is a complemented element and $A^{k(*+)}=A^{(k+1)(*+)}$ is obtained in contradiction of the choice of $A$. Therefore $B_{k} \neq \varnothing$ for every $k \geqslant 1$.

Clearly $B_{k} \subseteq D_{k}(A) \backslash D_{k-1}(A)$; so that $C_{k}=D_{k}\left(B_{k}\right) \cap A$ is a nonvoid closed set. For any $c \in C_{k}$, there exists a set $\left\{x_{0}, \ldots, x_{2 k}\right\} \subseteq P(L)$ such that $x_{2 i} \leqslant x_{2 i+1} \geqslant x_{2 i+2}$ for $i \in\{0, \ldots, k-1\}, x_{0}=c$, and $x_{2 k} \in B_{k}$. Clearly, $x_{2 j} \in B_{j}$ whenever $j \leqslant k$ and, consequently, $c \in C_{j}$. Therefore any finite subfamily of $\left\{C_{k}: k \geqslant 1\right\}$ has a nonvoid intersection; since $P(L)$ is compact, there exists some $a \in \bigcap\left\{C_{k}: k \geqslant 1\right\}$. Consequently, for every $k \geqslant 1$, the component $C(a)$ intersects $B_{k}$. Since $\left\{A^{k(*+)}: k \geqslant 1\right\}$ is an open covering of $C(a)$ and $B_{k+1} \cap A^{k(*+)}=\varnothing$, the component $C(a)$ cannot be closed.

Recall that a double p-algebra $A$ is regular if $a^{+}=b^{+}$and $a^{*}=b^{*}$ hold for $a, b \in A$ only when $a=b$; this is the case if and only if $a \wedge a^{+} \leqslant b \vee b^{*}$ for all $a, b \in A$.

Lemma 2.5 (Varlet [12]). A dp-space $(X, \tau, \leqslant)$ is the Priestley dual of a regular algebra if and only if $\operatorname{Ext}(X)=X$.

We conclude the preliminaries with a simple observation on dp-maps between duals of regular algebras.

Lemma 2.6. If $X_{i}$ are nonvoid connected posets satisfying $\operatorname{Ext}\left(X_{i}\right)=X_{i}$ for $i=0,1$ then any order preserving mapping $f: X_{0} \rightarrow X_{1}$ satisfying $f(\operatorname{Ext}(x))=\operatorname{Ext}(f(x))$ for all $x \in X_{0}$ is surjective.

Proof. Let $x \in f\left(X_{0}\right)$ be arbitrary. For any $y \in X_{1}$, there exists a one-to-one sequence $x=x_{0}, x_{1}, \ldots, x_{n}=y$ such that $x_{i}$ is comparable to $x_{i+1}$ for $i=0,1, \ldots, n$. If $x_{i} \in f\left(X_{0}\right)$ then $x_{i}=f(z)$ for some $z \in X_{0}$; but then $x_{i+1} \in \operatorname{Ext}\left(x_{i}\right)=\operatorname{Ext}(f(z))=f(\operatorname{Ext}(z)) \subseteq f\left(X_{0}\right)$. A simple induction yields $y \in f\left(X_{0}\right)$.

3. Regular varieties of bounded range. To show that no regular (quasi-)variety $\mathbf{V}$ of bounded range is universal, we exhibit a finite monoid which is not isomorphic to the endomorphism monoid of any regular algebra of bounded range.

Let $M$ be the freest monoid generated by $\{f, g\}$ in which $f$ is a left zero and $g$ is an invertible element of order three; that is, $M=\left\{\right.$ id, $\left.g, g^{2}, f, g f, g^{2} f\right\}$, and $f m=f$ for all $m \in M$. We aim to prove that $M$ cannot be isomorphic to the monoid of all dp-maps $h: Q \rightarrow Q$ for any dp-space $Q=(X, \tau, \leqslant)$ whose order components are all closed and which satisfies $\operatorname{Ext}(X)=X$. In view of $2.1,2.3,2.4$ and 2.5, this will show that the dual of $M$ is not isomorphic to the endomorphism monoid of any regular distributive double p-algebra of bounded range. More precisely, assuming that $M$ is contained in the monoid $\operatorname{End}(Q)$ of all dp-maps of $Q$ into itself, we propose to show that $\operatorname{End}(Q)$ contains an invertible element of order two. 
If $M$ is a submonoid of $\operatorname{End}(Q)$ then there exists some $a \in Q$ for which $g(f(a)) \neq$ $f(a)$; let $C$ denote the order component of $Q$ containing $b=f(a)$. From $f^{2}=f$, it follows that $f(b)=b$ and, by 2.6 , the image of the restriction $f \mid C$ of the dp-map $f$ is the whole component $C$; since $f$ is idempotent, $f(c)=c$ for all $c \in C$.

The image $g(C)$ of $C$ is another order component of $Q$. If $g(C)=C$ then $g(b) \in C$ and, since $f$ is the identity on $C$, we obtain $g(b)=f(g(b))=f(b)=b$, which contradicts the choice of $b \in Q$. Therefore $g(C) \cap C=\varnothing$ and, because $g$ is an automorphism of order three, the components $C, g(C), g^{2}(C)$ are pairwise disjoint.

Since these three components are closed in $Q$, from 2.2 , we obtain a clopen decreasing set $A$ such that $C \subseteq A$ and $g(C) \cup g^{2}(C) \subseteq X \backslash A$. If the range of the double p-algebra dual to $Q$ is $n$ then the clopen set $B=A^{n(+*)} \subseteq A$ is a union of order components of $Q$ which includes the component $C$. Thus the set $D=B \backslash\left(g(B) \cup g^{2}(B)\right)$ is a clopen union of order components of $Q$, and the sets $D, g(D), g^{2}(D)$ are pairwise disjoint; if $i, j \in\{0,1,2\}$ then $g^{i}(C) \subseteq g^{j}(D)$ just when $i=j$.

Define a mapping $h: Q \rightarrow Q$ by $h \uparrow D=g \uparrow D, h \uparrow g(D)=g^{2} \uparrow g(D)$ and $h(x)=x$ for $x \in X \backslash(D \cup g(D))$. Since the three sets $D, g(D)$ and $X \backslash(D \cup g(D))$ are unions of components of $Q$ and form a clopen decomposition of $Q$, and because $g$ is a dp-map, the mapping $h$ is continuous and satisfies $h(\operatorname{Ext}(x))=\operatorname{Ext}(h(x))$ for all $x \in X$. Clearly, $h^{2}(x)=x$ for all $x \in X$. Altogether, $h$ is an involutory dp-map of $Q$ onto $Q$.

This completes the proof of Theorem 1.2.

4. The variety of regular algebras. For every $n \geqslant 3$, define an ordered space $A_{n}$ on the set $2 n=\{0,1, \ldots, 2 n-1\}$ by

$$
a_{2 i} \leqslant a_{2 i \oplus 1} \geqslant a_{2 i \oplus 2} \text { for all } i \in n,
$$

where $\oplus$ denotes addition modulo $2 n$. Thus $A_{n}$ is an $n$-crown with $\operatorname{Min}\left(A_{n}\right)=\left\{a_{2 i}: i \in n\right\}$ and $\operatorname{Max}\left(A_{n}\right)=\left\{a_{2 i \oplus 1}: i \in n\right\}$. Equipped with the discrete topology, $A_{n}$ is a dp-space of a regular algebra.

Let $\mathbb{Z}$ be the set of all integers and let $B=\left\{b_{j}: j \in \mathbb{Z}\right\}$ be ordered so that

$$
\begin{aligned}
& b_{2 i} \leqslant b_{2 i+1} \geqslant b_{2 i+2} \text { for all } i \in \mathbb{Z} \text {, and } \\
& b_{0} \leqslant b_{3} .
\end{aligned}
$$

The connected poset $(B, \leqslant)$ is the disjoint union of $\operatorname{Max}(B)=\left\{b_{2 i+1}: i \in \mathbb{Z}\right\}$ with $\operatorname{Min}(B)=\left\{b_{2 i}: i \in \mathbb{Z}\right\}$.

LEMMA 4.1. Let $f$ be an order preserving mapping such that $f(\operatorname{Ext}(x))=\operatorname{Ext}(f(x))$ for every $x$ in the domain of $f$. Then:

(a) if $f: A_{m} \rightarrow A_{n}$ then $n$ divides $m$,

(b) if $f: B \rightarrow B$ then $f=\mathrm{id}_{B}$.

Proof. Let $f: A_{m} \rightarrow A_{n}$ be such a mapping; since it is onto by 2.6 , we have $m \geqslant n$. Having renumbered elements of $A_{n}$ as needed, we may assume that $f\left(a_{0}\right)=a_{0}$ and $f\left(a_{1}\right)=a_{1}$. Since $\operatorname{Min}\left(a_{1}\right)=\left\{a_{0}, a_{2}\right\}$ in both posets, we have $f\left(a_{0}\right)=a_{0}$ and $f\left(a_{2}\right)=a_{2}$. However, $\operatorname{Max}\left(a_{2}\right)=\left\{a_{1}, a_{3}\right\}$ in either poset again, and $f\left(a_{3}\right)=a_{3}$ follows. Continuing inductively, we find that $f\left(a_{k}\right)=a_{r(k)}$ for all $k \in 2 m$, where $r(k)$ is the remainder of $k(\bmod 2 n)$. Therefore $2 m \equiv 0(\bmod 2 n)$, that is, $n$ divides $m$ and $(a)$ is proved. 
To demonstrate (b), assume $f: B \rightarrow B$. Then $f$ is onto by 2.6. Since $\operatorname{Min}(b)$ has more than two elements only when $b=b_{3}$, it follows that $f\left(\operatorname{Max}(B) \backslash\left\{b_{3}\right\}\right) \subseteq \operatorname{Max}(B) \backslash\left\{b_{3}\right\}$ and hence $f\left(b_{3}\right)=b_{3}$; dually, $f\left(\operatorname{Min}(B) \backslash\left\{b_{0}\right\}\right) \subseteq \operatorname{Min}(B) \backslash\left\{b_{0}\right\}$ and $f\left(b_{0}\right)=b_{0}$.

From $b_{0} \leqslant b_{1} \geqslant b_{2} \leqslant b_{3}$, we thus obtain $b_{0} \leqslant f\left(b_{1}\right) \geqslant f\left(b_{2}\right) \leqslant b_{3}$. These requirements are satisfied only when $f\left(b_{1}\right)=b_{1}$ and $f\left(b_{2}\right)=b_{2}$. Therefore $\left\{b_{0}, b_{2}, f\left(b_{4}\right)\right\}=$ $f\left(\operatorname{Min}\left(b_{3}\right)\right)=\operatorname{Min}\left(b_{3}\right)=\left\{b_{0}, b_{2}, b_{4}\right\}$, and $f\left(b_{4}\right)=b_{4}$ follows. Hence $\left\{b_{3}, f\left(b_{5}\right)\right\}=$ $\operatorname{Max}\left(b_{4}\right)=\left\{b_{3}, b_{5}\right\}$ yields $f\left(b_{5}\right)=b_{5}$. Continuing inductively for $k \geqslant 6$, we conclude that $f\left(b_{j}\right)=b_{j}$ for all $j \geqslant 0$. An analogous argument shows that $f\left(b_{j}\right)=b_{j}$ also for $j \leqslant-1$.

The posets described above will be used to construct a full embedding of the category $\mathbf{L}(2)$ of topologized $(0,1)$-lattices with two unary operations [3] into the category of Priestley spaces dual to algebras from $\mathbf{R}$.

Following [3], $(X, \tau, \vee, \wedge, \alpha, \beta, 0,1)$ is an object of the category $\mathbf{L}(2)$ if and only if

$(X, \tau)$ is a compact totally disconnected space,

$(X, \vee, \wedge, 0,1)$ is a $(0,1)$-lattice,

$\alpha, \beta: X \rightarrow X$ are continuous mappings,

$\vee, \wedge: X^{2} \rightarrow X$ are continuous with respect to the product topology of $X^{2}$.

The class of morphisms of $\mathbf{L}(2)$ is formed by all lattice $(0,1)$-homomorphisms that are continuous with respect to the given Stone topology and preserve the unary operations $\alpha$ and $\beta$.

The fourth section of [3] demonstrates the universality of the category $\mathbf{L}(2)^{\text {opp }}$ dually isomorphic to the category $\mathbf{L}(2)$. To use this fact in proving the universality of the variety $\mathbf{R}$ of regular distributive double p-algebras, we need to construct a full embedding $\Phi$ of the category $\mathbf{L}(2)$ into the category of all dp-maps between dp-spaces dual to members of $\mathbf{R}$.

For any object $L=(X, \tau, \vee, \wedge, \alpha, \beta, 0,1)$ of $\mathbf{L}(2)$, the underlying poset $F(L)$ of $\Phi(L)$ will be the disjoint union of suitably assigned copies of posets $A_{n}$ and $B$.

For notational convenience, for each $j \in\{1, \ldots, 8\}$, let $P_{j}: X^{2} \rightarrow X$ denote the map defined for all $(x, y) \in X^{2}$ as follows.

$$
\begin{array}{ll}
P_{1}(x, y)=x, & P_{2}(x, y)=y, \\
P_{3}(x, y)=x \vee y, & P_{4}(x, y)=x \wedge y, \\
P_{5}(x, y)=\alpha(x), & P_{6}(x, y)=\beta(y), \quad \text { and } \\
P_{7}(x, y)=0, & P_{8}(x, y)=1 .
\end{array}
$$

The continuity of all operations of $\mathbf{L}(2)$ implies that, for every $j \in\{1, \ldots, 8\}$, the set $P_{j}^{-1}\left(X^{\prime}\right)$ is open (resp. closed) in $X^{2}$ whenever $X^{\prime} \subseteq X$ is open (resp. closed).

Next we define the ordered space $\Phi(L)$ corresponding to an arbitrary object $L \in \mathbf{L}(2)$.

For each $i \in 10=\{0,1, \ldots, 9\}$, let $A(i)$ be the $p(i)$-crown $A_{p(i)}$ selected so that $p(i)$ are pairwise different odd primes. In what follows, the union $X^{2} \cup X$ is assumed to be disjoint.

Let $F(L)=F_{A}(L) \cup F_{B}(L)$, where

$$
\begin{aligned}
& F_{A}(L)=\bigcup\{X \times\{i\} \times A(i): i=0,1, \ldots, 8\} \cup X^{2} \times\{9\} \times A(9), \\
& F_{B}(L)=\left(X^{2} \cup X\right) \times\{1, \ldots, 8\} \times B .
\end{aligned}
$$


The partial order $\leqslant$ of $F(L)$ is that directly inherited from the posets $A(i)$ and $B$; that is, $p \leqslant q$ in $F(L)$ if and only if $p=(z, i, c), q=(z, i, d)$ and $c \leqslant d$ in $B$, or $c \leqslant d$ in one of the crowns $A(i)$ with $i \in\{0,1, \ldots, 9\}$. Hence every order component of $F(L)$ is order isomorphic to the countable poset $B$ or to one of the selected prime crowns $A(i)$.

The space $F_{A}(L)$ is thus the disjoint union of nine copies of the compact space $X$ and a single copy of the space $X^{2}$ compact in the product topology; therefore $F_{A}(L)$ is compact. Our intention is to conserve this compact topology $\rho$ of $F_{A}(L)$, and extend it to $F(L)$ in a manner which reflects the action of the operations of $L$. Note that the subspace $F_{B}(L)$, a disjoint union of countably many copies of the compact space $X^{2} \cup X$, is locally compact.

For any $i \in 10$ and each $a=a_{k} \in A(i)$, define

$$
B(i, a)=\left\{b_{q} \in B: q \equiv k(\bmod 2 p(i))\right\}
$$

and, for every $n \in \mathbb{Z}$, set

$$
\begin{array}{ll}
B(\uparrow n)=\left\{b_{q}: q \geqslant n\right\}, & B(\uparrow n, i, a)=B(\uparrow n) \cap B(i, a), \\
B(\downarrow n)=\left\{b_{q}: q \leqslant n\right\}, & B(\downarrow n, i, a)=B(\downarrow n) \cap B(i, a) .
\end{array}
$$

Clearly,

$$
B(\uparrow n)=\bigcup\{B(\uparrow n, i, a): a \in A(i)\} \quad \text { and } \quad B(\downarrow n)=\bigcup\{B(\downarrow n, i, a): a \in A(i)\} .
$$

For any given $i \in 10$, the sets $B(i, a) \subseteq B$ are unbounded and form a decomposition of $B$. In fact, the mapping $\gamma_{i}: B \rightarrow A(i)$ defined by

$$
\gamma_{i}^{-1}\{a\}=B(i, a) \text { for all } a \in A(i)
$$

is easily seen to satisfy $\gamma_{i}(\operatorname{Ext}(b))=\operatorname{Ext}\left(\gamma_{i}(b)\right)$ for all $b \in B \backslash\left\{b_{0}, b_{3}\right\}$; the comparability $b_{0} \leqslant b_{3}$ constitutes the only reason why $\gamma_{i}$ fails to satisfy $\gamma_{i}(\operatorname{Ext}(b))=\operatorname{Ext}\left(\gamma_{i}(b)\right)$ for all $b \in B$.

Further, for any subset $C$ of $F(L)$, each $i \in\{0, \ldots, 8\}$, and any $a \in A(i)$, define

$$
X(C, i, a)=\{x \in X:(x, i, a) \in C\}
$$

analogously, for any $a \in A(9)$, set

$$
X^{2}(C, 9, a)=\left\{(x, y) \in X^{2}:((x, y), 9, a) \in C\right\} .
$$

Finally, for $b \in B$ and $j \in\{1, \ldots, 8\}$, define

$$
\begin{aligned}
X(C, j, b) & =\{x \in X:(x, j, b) \in C\}, \text { and } \\
X^{2}(C, j, b) & =\left\{(x, y) \in X^{2}:((x, y), j, b) \in C\right\} .
\end{aligned}
$$

The topology $\sigma$ of $F(L)$ is defined by the requirement that its open basis consist of all $C \subseteq F(L)$ satisfying the conditions (a)-(f) listed below.

(a) $X(C, i, a)$ is open in $X$ for all $a \in A(i)$ with $i \in\{0,1, \ldots, 8\}$, and $X^{2}(C, 9, a)$ is open in $X^{2}$ for all $a \in A(9)$,

(b) $X(C, j, b)$ is open in $X$ and $X^{2}(C, j, b)$ is open in $X^{2}$ for every $j \in\{1, \ldots, 8\}$ and every $b \in B$, 
(c) for every $j \in\{1, \ldots, 8\}$ and each $a \in A(0)$, there is some $n \in \mathbb{Z}$ such that $X(C, 0, a) \times\{j\} \times B(\downarrow n, 0, a) \subseteq C$,

(d) for every $j \in\{1, \ldots, 8\}$ and each $a \in A(j)$, there is some $m \in \mathbb{Z}$ such that $X(C, j, a) \times\{j\} \times B(\uparrow m, j, a) \subseteq C$

(e) for every $j \in\{1, \ldots, 8\}$ and each $a \in A(j)$, there is some $m \in \mathbb{Z}$ such that $P_{j}^{-1}(X(C, j, a)) \times\{j\} \times B(\uparrow m, j, a) \subseteq C$,

(f) for every $j \in\{1, \ldots, 8\}$ and each $a \in A(9)$, there is some $n \in \mathbb{Z}$ such that $X^{2}(C, 9, a) \times\{j\} \times B(\downarrow n, 9, a) \subseteq C$.

Since all properties (a)-(f) are satisfied by $C \cap C^{\prime}$ whenever $C$ and $C^{\prime}$ satisfy them, the topology $\sigma$ on $F(L)$ is well-defined. Also, $F_{B}(L)$ is $\sigma$-open, while $F_{A}(L)$ is a compact subspace of $F(L)$ because it is a (disjoint) union of finitely many compact spaces.

Let $k_{0}<0$ and $k_{1}>3$ be integers. It is routine to verify that, for any clopen $X^{\prime} \subseteq X$ and any clopen $Y \subseteq X^{2}$, the following subsets of $F(L)$ with $p \leqslant k_{0}, m, n \geqslant k_{1}$, and $j \in\{1, \ldots, 8\}$, are $\sigma$-clopen and form an open basis $S\left(k_{0}, k_{1}\right)$ of $\sigma$ :

$$
\begin{aligned}
& X^{\prime} \times\{j\} \times\{b\} \text { and } Y \times\{j\} \times\{b\} \text { for any } b \in B, \\
& X^{\prime} \times\{j\} \times(\{a\} \cup B(\uparrow m, j, a)) \cup P_{j}^{-1}\left(X^{\prime}\right) \times\{j\} \times B(\uparrow n, j, a) \text { for } a \in A(j), \\
& X^{\prime} \times\{0\} \times\{a\} \cup X^{\prime} \times\{1, \ldots, 8\} \times B(\downarrow n, 0, a) \text { for } a \in A(0), \\
& Y \times\{9\} \times\{a\} \cup Y \times\{1, \ldots, 8\} \times B(\downarrow n, 9, a) \text { for } a \in A(9) .
\end{aligned}
$$

LEMMA 4.2. Let $\operatorname{cl}(W)$ denote the $\sigma$-closure of $W \subseteq F(L)$, and let $\delta(W)=\operatorname{cl}(W) \backslash W$. Then the following clauses list $\delta(W)$ for any $n \in \mathbb{Z}, j \in\{1, \ldots, 8\}, x \in X$, and $(x, y) \in X^{2}$.

(A) $\delta(W)=\{(x, 0, a)\}$ when $W=\{x\} \times\{j\} \times B(\downarrow n, 0, a)$ and $a \in A(0)$,

(B) $\delta(W)=\{(x, j, a)\}$ when $W=\{x\} \times\{j\} \times B(\uparrow n, j, a)$ and $a \in A(j)$,

(C) $\delta(W)=\left\{\left(P_{j}(x, y), j, a\right)\right\}$ when $W=\{(x, y)\} \times\{j\} \times B(\uparrow n, j, a)$ and $a \in A(j)$,

(D) $\delta(W)=\{((x, y), 9, a)\}$ when $W=\{(x, y)\} \times\{j\} \times B(\downarrow n, 9, a)$ and $a \in A(9)$.

Proof. Straightforward from (a)-(f).

Lemma 4.3. The ordered space $\Phi(L)=(F(L), \sigma, \leqslant)$ is compact and totally order disconnected.

Proof. To demonstrate compactness, assume $\Gamma$ to be a covering of $\Phi(L)$ by basic open sets. Since $F_{A}(L)$ is compact in $\sigma$, it follows that $F_{A}(L) \subseteq \bigcup \Gamma^{\prime}=D$ for some finite $\left\{C_{p}: p \in P\right\}=\Gamma^{\prime} \subseteq \Gamma$.

In particular, $X \times\{0\} \times A(0) \subseteq D$. Since every $C_{p}$ satisfies (c), for each $j \in$ $\{1, \ldots, 8\}$ and $a \in A(0)$, there exist $p \in P$ and $n=n(j, a, p)$ such that $X\left(C_{p}, 0, a\right) \times$ $\{j\} \times B(\downarrow n, 0, a) \subseteq C_{p}$. Thus if $n(0)$ is the least of (the finitely many) such integers $n(j, a, p)$ then $X \times\{1, \ldots, 8\} \times B(\downarrow n(0)) \subseteq D$.

Analogously, $X \times\{j\} \times A(j) \subseteq D$ for each $j \in\{1, \ldots, 8\}$. Using (d) instead of (c) yields integers $m(j)$ for which $X \times\{j\} \times B(\uparrow m(j)) \subseteq D$. Since the complement $F_{j}$ of any $B(\downarrow n(0)) \cup B(\uparrow m(j))$ in $B$ is finite, $(X \times\{j\} \times B) \backslash D$ is contained in the compact set $X \times\{j\} \times F_{j}$ for every $j \in\{1, \ldots, 8\}$.

Observing that $P_{j}^{-1}(X)=X^{2}$, from $X \times\{j\} \times A(j) \subseteq D$, and using (e) this time, we similarly produce integers $m(j)$ for which $X^{2} \times\{j\} \times B(\uparrow m(j)) \subseteq D$. Since $X^{2} \times\{9\} \times$ $A(9) \subseteq D$, the condition (f) provides integers $n(j)$ for which $X^{2} \times\{j\} \times B(\downarrow n(j)) \subseteq D$. Just as before, the set $\left(X^{2} \times\{1, \ldots, 8\} \times B\right) \backslash D$ is contained in a compact subset of $F(L)$. 
Thus, in each case, the set $F(L) \backslash D$ is contained in a compact subset of $F(L)$, and the compactness of $\Phi(L)$ follows.

To show that $\Phi(L)$ is totally order disconnected, for any $p \neq q$ in $F(L)$, we must find a clopen increasing set $P \subseteq F(L)$ such that $p \in P$ and $q \in F(L) \backslash P$.

If $p \in F_{B}(L)$ then $p=(z, j, b)$ for some $z \in X^{2} \cup X, j \in\{1, \ldots, 8\}$ and $b \in B$. Since $[b]$ is finite, for any clopen $Z \subseteq X^{2} \cup X$ containing $z$, the set $P(Z)=Z \times\{j\} \times[b]$ is clopen and increasing, and it separates $p$ from all $q \in F_{A}(L)$. Any other $q \in F(L)$ has the form $q=\left(z^{\prime}, j^{\prime}, b^{\prime}\right) \in\left(X^{2} \cup X\right) \times\{1, \ldots, 8\} \times B$, and it lies in $P(Z)$ only when $z^{\prime} \in Z, j^{\prime}=j$ and $b^{\prime} \geqslant b$. But then $z^{\prime} \neq z$ follows from $p \neq q$. Since $X^{2} \cup X$ is totally disconnected, there is a clopen $Z^{\prime} \subseteq X^{2} \cup X$ containing $z$ but not $z^{\prime}$, so that $P\left(Z^{\prime}\right)$ separates $p$ from $q$ again.

A dual argument shows the separation property also when $q \in F_{B}(L)$; hence we may assume that $p, q \in F(L) \backslash F_{B}(L)=F_{A}(L)$. To complete the proof, for every $p \in F_{A}(L)$, we must exhibit a clopen increasing set $P$ such that $q \notin P \cap F_{A}(L)$.

Let $p=(z, 0, a) \in X \times\{0\} \times A(0)$, and let $Y \subseteq X$ be clopen in $X$ and such that $z \in Y$. For any $a^{\prime} \geqslant a$ and $n \leqslant-1$, the set

$$
P\left(Y, a^{\prime}\right)=Y \times\{0\} \times\left\{a^{\prime}\right\} \cup Y \times\{1, \ldots, 8\} \times B\left(\downarrow n, 0, a^{\prime}\right)
$$

is clopen. Then $P(Y)=\bigcup\left\{P\left(Y, a^{\prime}\right): a^{\prime} \in[a)\right\}$ is clopen since $[a)$ is finite, and it is increasing because $n \leqslant-1$; clearly $p \in P(Y)$. If $q \in P(Y)$ then $q=\left(z^{\prime}, 0, a^{\prime}\right)$ for some $a^{\prime} \in[a)$; hence $z^{\prime} \neq z$ by the hypothesis. Since $X$ is totally disconnected, there exists a clopen set $Y^{\prime} \subseteq X$ containing $z$ but not $z^{\prime}$. The clopen increasing set $P\left(Y^{\prime}\right)$ then separates $p$ from $q$ as was to be shown. With $X$ replaced by $X^{2}$, this argument disposes of the case of any $p=(z, 9, a) \in X^{2} \times\{9\} \times A(9)$.

Finally, let $p=(x, j, a) \in X \times\{j\} \times A(j)$ for some $j \in\{1, \ldots, 8\}$. Choose any $m \geqslant 1$; for each $a^{\prime} \geqslant a$ and $X^{\prime} \subseteq X$ clopen in $X$, set

$$
P\left(X^{\prime}, a^{\prime}\right)=X^{\prime} \times\{j\} \times\left\{a^{\prime}\right\} \cup\left(X^{\prime} \cup P_{j}^{-1}\left(X^{\prime}\right)\right) \times\{j\} \times B\left(\uparrow m, j, a^{\prime}\right) .
$$

Each $P\left(X^{\prime}, a^{\prime}\right)$ is clopen, and $P\left(X^{\prime}\right)=\bigcup\left\{P\left(X^{\prime}, a^{\prime}\right): a^{\prime} \in[a)\right\}$ is a clopen increasing set containing $p$. If $q \in P\left(X^{\prime}\right)$ then $q=\left(x^{\prime}, j, a^{\prime}\right)$ for some $x \in X^{\prime}$; from $p \neq q$, it follows that $x \neq x^{\prime}$ in $X$. Hence $x \in X^{\prime \prime}$ and $x^{\prime} \in W \backslash X^{\prime \prime}$ for some $X^{\prime \prime}$ clopen in $X$, and the set $P\left(X^{\prime \prime}\right)$ separates $p$ from $q$ as required.

\section{Lemma 4.4. The Priestley space $\Phi(L)$ represents a regular double p-algebra.}

Proof. In view of 2.5 and 4.3 , it suffices to prove that $(C]$ and $[C)$ are $\sigma$-clopen for any $\sigma$-clopen $C \subseteq F(L)$; all arguments may be restricted to members of the basis $S\left(k_{0}, k_{1}\right)$ with $k_{0}<-1$ and $k_{1}>4$.

If $C=X^{\prime} \times\{j\} \times\{b\}$ for some clopen $X^{\prime} \subseteq X, j \in\{1, \ldots, 8\}$, and some $b \in B$ then either $b$ is minimal and $(C]=C$, or $b=b_{2 k+1}$ is maximal and $(C] \backslash C$ is the union of clopen sets $X^{\prime} \times\{j\} \times\left\{b_{2 k}\right\}$ and $X^{\prime} \times\{j\} \times\left\{b_{2 k+2}\right\}$ from $S\left(k_{0}, k_{1}\right)$.

If $C=X^{\prime} \times\{j\} \times(\{a\} \cup B(\uparrow m, j, a)) \cup P_{j}^{-1}\left(X^{\prime}\right) \times\{j\} \times B(\uparrow n, j, a)$ for some clopen $X^{\prime} \subseteq X, j \in\{1, \ldots, 8\}, a \in A(j)$, and $m, n \geqslant 4$ then $(C]=C$ whenever $a$ is minimal.

Otherwise $(C] \backslash C$ is the union of two clopen sets of the form

$$
X^{\prime} \times\{j\} \times\left(\left\{a^{\prime}\right\} \cup B\left(\uparrow m^{\prime}, j, a^{\prime}\right)\right) \cup P_{j}^{-1}\left(X^{\prime}\right) \times\{j\} \times B\left(\uparrow n^{\prime}, j, a^{\prime}\right),
$$

where $a^{\prime}<a$ in $A(j)$, and $m^{\prime}, n^{\prime} \in B\left(j, a^{\prime}\right)$ are the least even integers respectively 
satisfying $m^{\prime} \geqslant m-1$ and $n^{\prime} \geqslant n-1$. Since these sets are members of the basis $S\left(k_{0}, k_{1}-1\right)$, the set $(C)$ is clopen.

Analogous arguments apply to members of the basis $S\left(k_{0}, k_{1}\right)$ of the remaining two types. Dualizing these arguments we easily show that $[C)$ is clopen for any $C \in$ $S\left(k_{0}, k_{1}\right)$.

For any morphism $f: L \rightarrow L^{\prime}$ of the category $\mathbf{L}(2)$, we now define a mapping $\Phi(f): \Phi(L) \rightarrow \Phi\left(L^{\prime}\right)$ as

$$
\begin{aligned}
& \Phi(f)(x, i, a)=(f(x), i, a) \text { for all } x \in X \text {, all } i \in\{0,1, \ldots, 8\}, \text { and all } a \in A(i), \\
& \Phi(f)(x, j, b)=(f(x), j, b) \text { for all } x \in X, \text { all } j \in\{1, \ldots, 8\}, \text { and all } b \in B, \\
& \Phi(f)((x, y), j, b)=((f(x), f(y)), j, b) \text { for all }(x, y) \in X^{2}, \text { all } j \in\{1, \ldots, 8\}, \text { and all } \\
& b \in B, \\
& \Phi(f)((x, y), 9, a)=((f(x), f(y)), 9, a) \text { for all }(x, y) \in X^{2} \text { and all } a \in A(9) .
\end{aligned}
$$

Since the effect of $\Phi(f)$ is confined only to the first component of each $(z, i, c) \in$ $F(L)$, and because the order of $F(L)$ is that inherited from the third component of its elements, the mapping $\Phi(f)$ is order preserving and satisfies $\operatorname{Ext}(\Phi(f)(t))=\Phi(f)(\operatorname{Ext}(t))$ for every $t \in F(L)$.

The continuity of the $\mathbf{L}(2)$-morphism $f$ on $X$ implies that $f \times f$ is continuous on $X^{2}$. Since the topology of the open subspace $F_{B}(L)$ of $\Phi(L)$ and of its complement $F_{A}(L)$ is that of the product of $X^{2} \cup X$ with a discrete space, the respective restrictions of $\Phi(f)$ to these two subspaces are continuous. To verify the continuity of $\Phi(f)$, it thus suffices to demonstrate that $\Phi(f)(\operatorname{cl}(U))$ is contained in $\operatorname{cl}(\Phi(f)(U))$ for any $U \subseteq F_{B}(L)$. This, however, is accomplished through a routine use of 4.2 and of the fact that $P_{j}(f(x), f(y))=f\left(P_{j}(x, y)\right)$ holds for all $(x, y) \in X^{2}$ and for each $j \in\{1, \ldots, 8\}$.

Finally, to show that $\Phi$ is a full embedding, suppose that $h: \Phi(L) \rightarrow \Phi\left(L^{\prime}\right)$ is a continuous mapping such that $\operatorname{Ext}(h(t))=h(\operatorname{Ext}(t))$ for all $t \in F(L)$.

Recall that each order component $C$ of $F(L)$ has the form $C=\{z\} \times\{i\} \times A(i) \cong$ $A_{p(i)}$ for some $i \in 10$, or $C=\{z\} \times\{j\} \times B \cong B$ for some $j \in\{1, \ldots, 8\}$ and $z \in X^{2} \cup X$. According to 2.6, $h(C)$ must be an order component of $F\left(L^{\prime}\right)$.

If $C=\{z\} \times\{i\} \times A(i)$ then $f(C)$ is a finite order component of $\Phi\left(L^{\prime}\right)$, that is, a component isomorphic to some $A(k)$. By 4.1(a) and the choice of the primes $p(i)$, this is possible only when $k=i$. Therefore $h(\{z\} \times\{i\} \times A(i))=\left\{z^{\prime}\right\} \times\{i\} \times A(i)$ for all $i \in 10$ and, in particular, $z^{\prime} \in X^{\prime}$ if and only if $z \in X$.

From 4.2 it easily follows that for all $j \in\{1, \ldots, 8\}, x \in X$ and $(x, y) \in X^{2}$,

$$
\begin{aligned}
& \delta(\{x\} \times\{j\} \times B)=\{x\} \times\{0\} \times A(0) \cup\{x\} \times\{j\} \times A(j), \text { and } \\
& \delta(\{(x, y)\} \times\{j\} \times B)=\left\{P_{j}(x, y)\right\} \times\{j\} \times A(j) \cup\{(x, y)\} \times\{9\} \times A(9) .
\end{aligned}
$$

Since $h$ is continuous, the closure of the order component $h(\{x\} \times\{j\} \times B)$ must contain $h(\{x\} \times\{0\} \times A(0)) \cong A(0)$ and $h(\{x\} \times\{j\} \times A(j)) \cong A(j)$. This is impossible when $h(\{x\} \times\{j\} \times B)$ is finite and, therefore, closed. Hence $h(\{x\} \times\{j\} \times B) \cong B$. By 4.1(b) and by the definition of $\Phi\left(L^{\prime}\right)$, for some $z^{\prime} \in\left(X^{\prime}\right)^{2} \cup X^{\prime}, k \in\{1, \ldots, 8\}$ and all $b \in B$ we must have $h(x, j, b)=\left(z^{\prime}, k, b\right)$. Using the continuity of $h$, from $4.2(\mathrm{~A})$ and $4.2(\mathrm{~B})$, we now obtain $k=j$, and the existence of a mapping $f: X \rightarrow X^{\prime}$ for which $h(x, j, b)=$ $(f(x), j, b)$ for all $x \in X, j \in\{1, \ldots, 8\}, b \in B$, and also $h(x, i, a)=(f(x), i, a)$ for all $x \in X, i \in\{0,1, \ldots, 8\}$, and all $a \in A(i)$. 
An analogous argument applied to the infinite component $h(\{(x, y)\} \times\{j\} \times B)$ implies the existence of a mapping $g: X^{2} \rightarrow\left(X^{\prime}\right)^{2}$ such that $h((x, y), j, b)=(g(x, y), j, b)$ for all $j \in\{1, \ldots, 8\}$ and $b \in B$, and also that $h((x, y), 9, a)=(g(x, y), 9, a)$ for all $a \in A(9)$.

Consequently, for all $(x, y) \in X^{2}$, all $j \in\{1, \ldots, 8\}$, and any $a \in A(j)$, the continuity of $h$ implies that $h\left(P_{j}(x, y), j, a\right)=\left(f\left(P_{j}(x, y)\right), j, a\right)$ is contained in the closure of $\{g(x, y)\} \times\{j\} \times B$. This, however, is possible only when $P_{j}(g(x, y))=f\left(P_{j}(x, y)\right)$.

From $P_{1}(x, y)=x$ and $P_{2}(x, y)=y$ it now follows that $g(x, y)=(f(x), f(y))$ for all $(x, y) \in X^{2}$. When $j=3$ or $j=4$, we obtain $f(x \vee y)=f\left(P_{3}(x, y)\right)=P_{3}(f(x), f(y))=$ $f(x) \vee f(y)$ and $f(x \wedge y)=f(x) \wedge f(y)$, respectively. Furthermore, $f(\alpha(x))=f\left(P_{5}(x, y)\right)=$ $P_{5}(f(x), f(y))=\alpha(f(x))$, and $f(\beta(y))=f\left(P_{6}(x, y)\right)=P_{6}(f(x), f(y))=\beta(f(y))$. Finally, $f(0)=f\left(P_{7}(x, y)\right)=P_{7}(f(x), f(y))=0$ and, similarly, $f(1)=1$.

Altogether, $f: L \rightarrow L^{\prime}$ is a continuous lattice $(0,1)$-homomorphism which preserves the two unary operations, and $h=\Phi(f)$ and was to be shown. Thus $\Phi$ is a full functor, and the proof of Theorem 1.1 is complete.

In conclusion, we sincerely thank Rodney Beazer for his comments on an earlier version of this paper and, particularly, for his useful suggestions concerning the variety of double Heyting algebras. In conjunction with the main result of [1], Theorem 4.5 below provides another natural example of an arithmetical, congruence uniform, yet categorically universal variety. A brief direct proof is included for the sake of completeness.

THEOREM 4.5 (R. Beazer). The variety of double Heyting algebras is categorically universal.

Proof. According to Priestley [10], the dual of the category of all double Heyting algebras is formed by all Priestley spaces in which $(C]$ and $[C)$ are clopen for any clopen convex subset $C$, and by all their dh-maps, that is, all continuous order-preserving maps satisfying $f(z]=(f(z)]$ and $f[z)=[f(z))$ for every element $z$ in the domain space of $f$.

Since every dp-space $Z=\Phi(L)$ constructed in this section is a disjoint union of clopen sets $\operatorname{Min}(Z)$ and $\operatorname{Max}(Z)$, for any clopen subset $C$ of $Z$, the sets $(C]=(C \cap \operatorname{Max}(Z)] \cup(C \cap \operatorname{Min}(Z))$ and $[C)=[C \cap \operatorname{Min}(Z)) \cup(C \cup \operatorname{Max}(Z))$ are clopen; thus every $\Phi(L)$ is the Priestley space of a double Heyting algebra as well.

The set of all dp-maps between such spaces coincides with that of all dh-maps. This becomes clear once it is noted that any dh-map preserves maximality and minimality, and that $(z]=\{z\}$ and $[z)=\{z\} \cup \operatorname{Max}(z)$ for any $z \in \operatorname{Min}(Z)$, while $(z]=\{z\} \cup \operatorname{Min}(z)$ and $[z)=\{z\}$ for every $z \in \operatorname{Max}(Z)$.

\section{REFERENCES}

1. M. E. Adams and R. Beazer, The variety of double Heyting algebras is congruence uniform, preprint.

2. M. E. Adams and R. Beazer, Congruence properties of distributive double p-algebras, preprint.

3. M. E. Adams, V. Koubek and J. Sichler, Homomorphisms and endomorphisms in varieties of pseudocomplemented distributive lattices (with applications to Heyting algebras), Trans. Amer. Math. Soc. 285 (1984), 57-79.

4. Z. Hedrlin and $J$ Sichler, Any boundable binding category contains a proper class of mutually disjoint copies of itself, Algebra Universalis 1 (1971), 97-103. 
5. T. Katriňák, The structure of distributive double p-algebras. Regularity and congruences, Algebra Universalis 3 (1973), 238-246. 195-219.

6. T. Katriňák, Subdirectly irreducible double p-algebras, Algebra Universalis 10 (1980),

7. V. Koubek and J. Sichler, Universal varieties of distributive double p-algebras, Glasgow J. Math. 26 (1985), 121-131.

8. H. A. Priestley, Representation of distributive lattices by means of ordered Stone spaces, Bull. London Math. Soc. 2 (1970), 186-190.

9. H. A. Priestley, Ordered topological spaces and the representation of distributive lattices, Proc. London Math. Soc. (3) 24 (1972), 507-530.

10. H. A. Priestley, Ordered sets and duality for distributive lattices, Ann. Discrete Math. 23 (1984), 36-90.

11. A. Pultr and V. Trnková, Combinatorial, algebraic and topological representations of groups, semigroups and categories (North-Holland, 1980).

12. J. C. Varlet, A regular variety of type $(2,2,1,1,0,0)$, Algebra Universalis 2 (1972), 218-223.

\section{MFF KU}

Malostranské nám. 25

11800 Praha 1

Czechoslovakia
UNIVERSITY OF MANITOBA

WINNIPEg, MANTTOBA

CANADA R3T 2N2 